Trinity College

Trinity College Digital Repository

Faculty Scholarship

2013

Femtosecond Photoelectron Point Projection Microscope

Erik Quinonez

Trinity College

Jonathan Handali

Trinity College

Brett Barwick

Trinity College, brett.barwick@trincoll.edu

Follow this and additional works at: https://digitalrepository.trincoll.edu/facpub

Part of the Physics Commons 


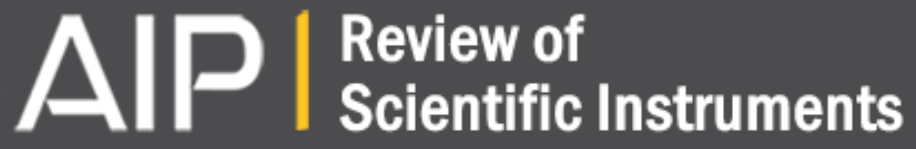

\section{Femtosecond photoelectron point projection microscope}

Erik Quinonez, Jonathan Handali, and Brett Barwick

Citation: Review of Scientific Instruments 84, 103710 (2013); doi: 10.1063/1.4827035

View online: http://dx.doi.org/10.1063/1.4827035

View Table of Contents: http://scitation.aip.org/content/aip/journal/rsi/84/10?ver=pdfcov

Published by the AIP Publishing

\section{GRANVILLE-PHILLIPS ${ }^{\circledR}$ Introducing the First}

advanced Vacuum Measurement Solutions

Vacuum Gauges:

Convectron", Micro-Ion, Stabil-Ion*,

Cold Cathode

Cold Cathode Gauge

worthy of the

Mass Spectrometers:

Vacuum Quality Monitors

Brooks

www.brooks.com

Granville-Phillips name!

-Unsurpassed Accuracy

-Predictive \& Easy Maintenance

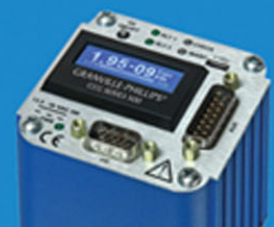




\title{
Femtosecond photoelectron point projection microscope
}

\author{
Erik Quinonez, Jonathan Handali, and Brett Barwick ${ }^{\text {a) }}$ \\ Department of Physics, Trinity College, 300 Summit St., Hartford, Connecticut 06106, USA
}

(Received 19 June 2013; accepted 12 October 2013; published online 30 October 2013)

\begin{abstract}
By utilizing a nanometer ultrafast electron source in a point projection microscope we demonstrate that images of nanoparticles with spatial resolutions of the order of 100 nanometers can be obtained. The duration of the emission process of the photoemitted electrons used to make images is shown to be of the order of $100 \mathrm{fs}$ using an autocorrelation technique. The compact geometry of this photoelectron point projection microscope does not preclude its use as a simple ultrafast electron microscope, and we use simple analytic models to estimate temporal resolutions that can be expected when using it as a pump-probe ultrafast electron microscope. These models show a significant increase in temporal resolution when comparing to ultrafast electron microscopes based on conventional designs. We also model the microscopes spectroscopic abilities to capture ultrafast phenomena such as the photon induced near field effect. ( 2013 AIP Publishing LLC. [http://dx.doi.org/10.1063/1.4827035]
\end{abstract}

\section{INTRODUCTION}

Recent advances have extended the domain of electron microscopy to include the ability to follow dynamics with femtosecond temporal resolution. It is now possible through these techniques to image processes such as melting, heating, mechanical vibrations, chemical modification, plasmon excitations, and fundamental physics phenomena with unprecedented resolutions in both space and time. ${ }^{1}$ This new field has been dubbed 4D ultrafast electron microscopy (UEM), ${ }^{1}$ and uses a femtosecond laser pulse to excite a specimen followed by a femtosecond electron pulse to image the ultrafast process. While UEM's have often been based on traditional transmission electron microscopes, ${ }^{1,2}$ alternative methods relying on projection imaging have been shown to be particularly useful for imaging plasmas and photoelectron bunches. ${ }^{3-6}$ Imaging with these techniques has been demonstrated with picosecond/femtosecond temporal resolution, however, their spatial resolution has been limited to the hundred $\mu \mathrm{m}$ level. ${ }^{3-6}$ In a complimentary technique, femtosecond photoelectron packets emitted from nanotips, which are doped with different materials, have been shown to provide spatial resolution on the nanometer level, though this technique as yet has not been used to follow ultrafast dynamics. ${ }^{7,8}$ The fact that the specimen is also the source of the photoelectrons used to create the images may make it difficult to convert this microscope to an UEM because both the pump and probe laser pulses would be incident on the specimen simultaneously. ${ }^{7,8}$

In this paper, we report the imaging capabilities of a femtosecond photoelectron point projection microscope which combines the techniques of electron point projection microscopy with an ultrafast field emission tip source. The microscope can be used to conduct low energy electron spectroscopy on arbitrary specimens, and when used as a UEM holds some advantages over traditional transmission electron microscopes based designs.

\footnotetext{
a) Author to whom correspondence should be addressed. Electronic mail:
} brett.barwick@trincoll.edu

\section{APPARATUS}

Our pulsed microscope relies on an ultrafast electron source that operates by focusing the output from a femtosecond oscillator onto a tungsten field emission tip. ${ }^{9-12}$ The emission time of the electrons created through this technique has been shown to be less than the duration of the initiating femtosecond laser pulse via autocorrelation ${ }^{9,11}$ and the emitted electron energy distributions demonstrate that the emission process can be as short as a single optical cycle. ${ }^{13,14}$ Combining this ultrafast field emission electron source with the technique of point projection electron microscopy demonstrates an alternative to the far more expensive and complicated conventional electron microscope platform.

A point projection microscope works by placing a field emission tip close to a specimen, where the magnification $M$ of the microscope ${ }^{15}$ is directly related to the ratio of the tip-to-specimen distance $d$, and the distance from the tip to detector $D$,

$$
M=\frac{D}{d} .
$$

As the distance $d$ is decreased (the specimen is brought closer to the tip) the magnification of the image increases.

A schematic of the femtosecond photoelectron point projection microscope is shown in Fig. 1. A field emission tip, with a diameter of the order of $\sim 100 \mathrm{~nm}$ is placed in front of a specimen/holder whose position can be moved relative to the tip by a 3 -axis motional feedthrough. The microscope is placed inside a vacuum system which provides minimal magnetic shielding (reducing the DC magnetic fields by $90 \%$, as compared to outside the chamber). We note, however, that previous experiments with a conventional point projection microscope that did not have magnetic shielding were still able to achieve nanometer resolution. ${ }^{15} \mathrm{~A}$ two dimensional single electron detector, consisting of a multichannel plate/phosphor screen, is located after the specimen and a CCD camera captures the images projected on the phosphor screen. Optical windows are located on the vacuum chamber to allow the 


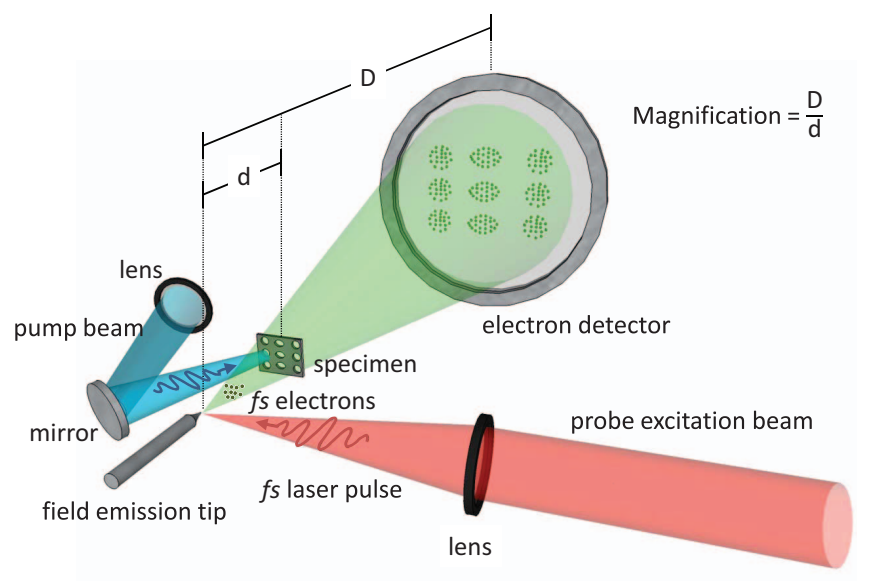

FIG. 1. Schematic of the femtosecond photoelectron point projection microscope. The pump beam is used to excite the sample, while the probe beam is used to generate the pulsed electron packets by excitation from a field emission tip. The electron packet is then accelerated to the sample and detected at a distance $\mathrm{D}$ from the source.

input of laser beams and a turbo-molecular pump is used to reach a pressure of $10^{-9}$ Torr after baking at $120^{\circ} \mathrm{C}$.

The microscope can be operated in a standard $\mathrm{CW}$ mode when the DC field applied to the tip is high enough to cause field emission of electrons. To create images utilizing pulsed femtosecond photoelectrons, the applied field is reduced so that negligible DC emission occurs (typically a $20 \%$ reduction is sufficient) and the tip is then irradiated with focused femtosecond laser pulses from a Ti:Sapphire oscillator. The laser outputs $800 \mathrm{~nm}$ pulses of $80 \mathrm{fs}$ duration at a repetition rate of $80 \mathrm{MHz}$ with pulse energies of $10 \mathrm{~nJ}$. The pulses are focused with a $40 \mathrm{~mm}$ focal length lens located inside the vacuum system resulting in a focal spot diameter of $\sim 10 \mu \mathrm{m}$. The lens is mounted on a 3-axis manipulator that allows it to be moved in relation to the tip. By moving the lens the focus is translated and the electron emission in pulsed operation mode is maximized.

The distance from the tip to the detector is fixed at $0.1 \mathrm{~m}$, however the distance from the tip-to-specimen, $d$, can be varied from $10^{-2} \mathrm{~m}$ down to $10^{-5} \mathrm{~m}$, which would correspond to magnifications of $10 \times$ to $10000 \times$. Resolutions down to the nanometer level are obtained using $10 \mathrm{k}$ magnification and are currently limited in our microscope by mechanical vibrations. The tip-to-specimen distance cannot be smaller than the radius of the laser focus at the tip because the specimen/holder begins to block the laser beam. This results in a reduction of photo-emitted electrons, as well as increasing the risk of incurring damage to the specimen and holder.

\section{RESULTS}

\section{A. Imaging capabilities}

To quantify the imaging capability of our electron microscope, we imaged $100 \mathrm{~nm}$ diameter silver nanowires (ACS Materials AgNWS-120 ethanol) which were deposited on a TEM grid (Quantifoil Multi A Micromachined Holey Carbon Grid). Images of the grid/nanowires taken with the

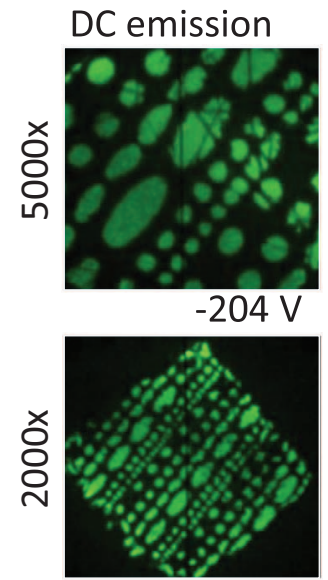

Photoelectrons

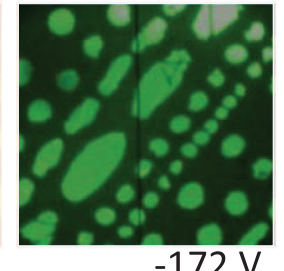

$-261 \mathrm{~V}$

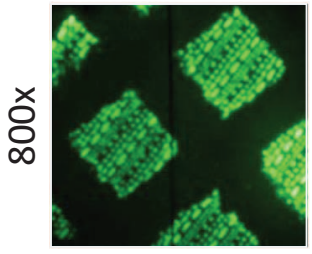

$-304 \mathrm{~V}$
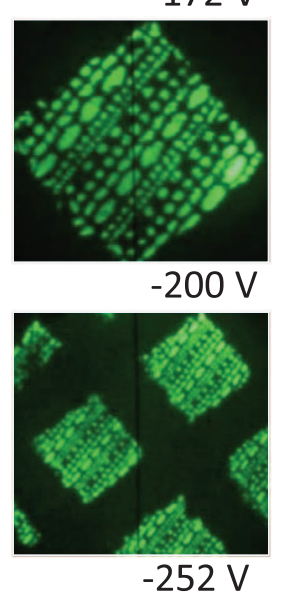

FIG. 2. Point projection images captured when operating the microscope with DC electron emission (left) and with femtosecond photoelectron packets (right). The smallest holes in the $5000 \times$ magnification image have a diameter of $1 \mu \mathrm{m}$ and the nanowires that become visible are $\sim 100 \mathrm{~nm}$ in diameter. The tip voltages (or kinetic electron energies at the specimen) are labeled for each image.

microscope in both $\mathrm{CW}$ mode (electrons being emitted from the tip due to DC field emission) and pulsed mode (electron emission being induced by $800 \mathrm{~nm}, 80 \mathrm{fs}$ laser pulses at $80 \mathrm{MHz}$ ) are shown in Fig. 2. Different magnifications are obtained by changing the tip-to-specimen distances. Images for both $\mathrm{CW}$ and pulsed mode are shown in Fig. 2. To achieve a magnification of $5000 \times$ in $\mathrm{CW}$ mode required tip voltage of $-204 \mathrm{~V}$, and for pulsed mode generation of photoelectrons a $-172 \mathrm{~V}$ tip voltage was used. For lower magnification, higher tip voltages were required; the corresponding tip voltages are listed in Fig. 2. All images use the same tip, all are $30 \mathrm{~s}$ exposures and the kinetic energy of the electrons at the sample is equal to $-e \mathrm{~V}_{\text {tip. At }}$. Ahe higher magnification of $5000 \times$ the smallest holes (diameter $1 \mu \mathrm{m}$ ) are clearly visible in both $\mathrm{CW}$ and pulsed modes and the silver nanowires (diameter $100 \mathrm{~nm}$ ) are easily visible in the $\mathrm{CW}$ mode and a few can be observed in pulsed mode (upper right of Fig. 2). Due to the relatively low kinetic energy of the electrons, some electrostatic lensing occurs near the specimen due to charging, which can change the image appearance at different voltages. These effects can be mitigated by careful specimen preparation and coating with materials of higher conductivity. The vertical dark line through all the images is due to a split phosphor screen that is used for electron coincident measurements and all the images were taken with electron pulses containing on average much less than one electron per packet.

An autocorrelation experiment was performed in order to investigate the temporal duration of the electron emission process from the tip, while in photoelectron imaging mode. ${ }^{9,11}$ By using the emission of electrons from the tip as a 
nonlinear detector in an interferometric autocorrelator, the emission process is shown to be of the order of the laser pulse duration when taken at $-238 \mathrm{~V}$ (negligible $\mathrm{CW}$ emission), see Fig. 3 (top panel). The autocorrelation trace is made by using an interferometer to create two laser pulses which are allowed to overlap. These two pulses incident on the tip constitute a pump-probe experiment and due to the highly nonlinear response of the emission process, if the first pulse affects the tip (by heating it up, for example) the tail of the signal would show this. However, no indication of tip modification is present in our data, indicating prompt emission. ${ }^{9}$ The autocorrelation data were taken as the images of the specimen were being observed on the $\mathrm{MCP} /$ phosphor detector. In addition to the autocorrelation result, we also investigated the polarization dependence of the electron emission and found that for laser polarizations parallel to the tip, the emission process is a maximum whereas polarizations perpendicular to the tip
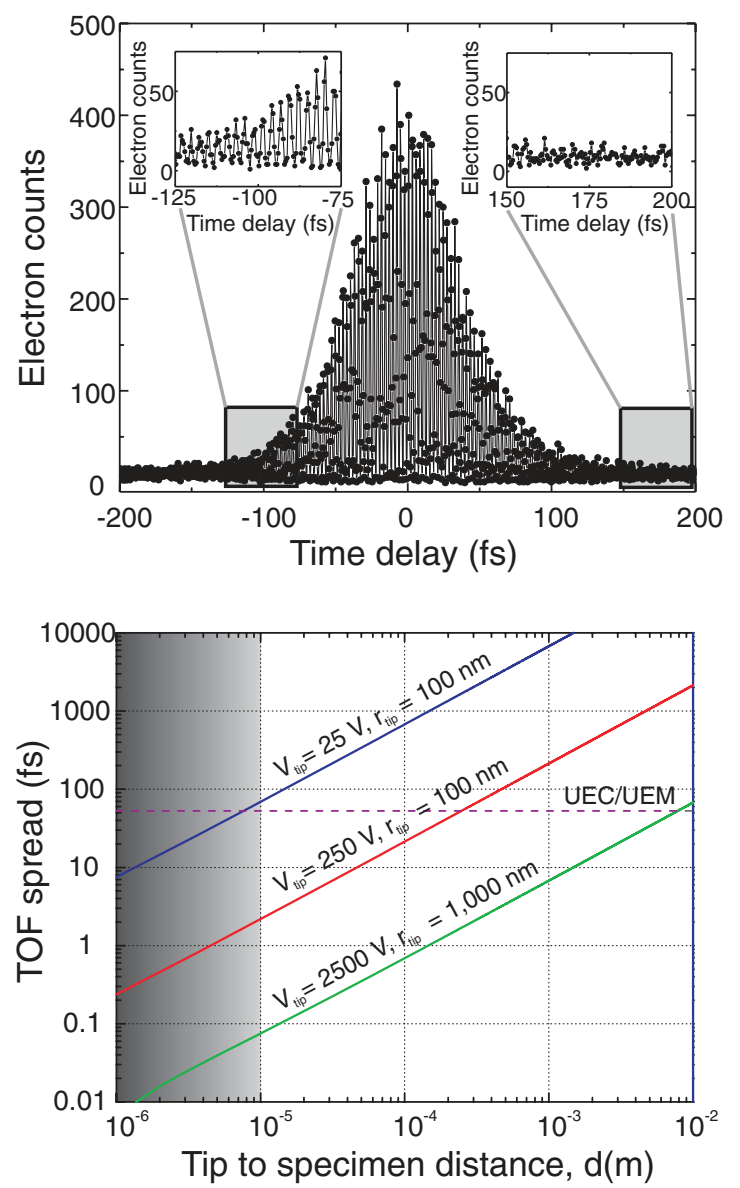

FIG. 3. Top panel: Autocorrelation trace using field emission tip as a nonlinear detector. Note the upper right inset shows a flat tail, indicating prompt electron emission. Bottom panel: Temporal spread in time-of-flight versus tip-to-specimen distance for electron packets with an initial energy spread of $\Delta \mathrm{E}=1 \mathrm{eV}$, for both UEC/UEM (Eq. (4)) and PPUEM (Eq. (2)) geometries. The UEC/UEM plot is generated using fixed values of $d_{\text {accel }}=3 \mathrm{~mm}$, $d_{\text {drift }}=0.645 \mathrm{~m}$, values taken from Ref. 20 , and an accelerating potential of $V_{\text {accel }}=60 \mathrm{kV}$. Neither $d_{\text {accel }}$ or $d_{\text {drift }}$ can be changed in a typical apparatus. The plots were created with $r_{\text {tip }}=100 \mathrm{~nm}$ and $V_{\text {accel }}$ of 25, 250 and $r_{\text {tip }}$ $=1000 \mathrm{~nm}$ for the $2500 \mathrm{eV}$ curve. The shaded experimental region indicates geometries that are decreasingly accessible while operating as a UEM as the tip-to-specimen region is decreased, caused by the photoelectron excitation pulse being blocked by the specimen. axis give no emission. ${ }^{9,10}$ These results confirm that the electron emission process is ultrafast in nature and occurs within $\sim 100$ fs for our setup, while operating in pulsed imaging mode.

\section{B. Time of flight spectroscopy in photoelectron mode}

In addition to imaging this microscope can be used as an electron energy spectrometer. Electron energy spectrometry is a powerful tool that can be used to measure the energy of the electron after it has interacted with a specimen. In a standard TEM, as the electrons travel through the specimen they can undergo inelastic collisions (loosing energy). These interactions include plasmon excitations, phonon excitations, and inner shell ionizations, just to name a few, which can be identified when looking at the spectroscopic data. For an ultrafast electron microscope, including one based on the device presented here, there is an additional interaction when the pump photon pulse interacts with the specimen that can result in both energy gains/losses when a photon is absorbed/emitted, and is the primary motivation for implementing a spectrometer in our microscope. To operate as a spectrometer, a time of flight technique is implemented. ${ }^{16}$ Our implementation can simultaneously collect spectroscopic data and images. When the femtosecond photoelectron pulses are emitted from the field emission tip, the laser sends a start signal to a time-toamplitude (TAC) converter. The emitted electrons are then accelerated in the tip-to-specimen region, which has a spatially nonlinear electric field due to the high curvature of the nanometer tip $^{17}$ that results in fields near the tip surface in excess of $10^{9} \mathrm{~V} / \mathrm{m}$. The resulting time of flight for an electron from the tip to the specimen can be approximated as

$$
T O F_{t i p}=d \sqrt{\frac{m_{e}}{2 e V_{t i p}}}\left[1+\frac{r_{t i p}}{2 d} \ln \left(\frac{d}{r_{t i p}}\right)\right],
$$

where $r_{\text {tip }}$ is the radius of the tip, $V_{\text {tip }}$ is the tip accelerating voltage (and also the kinetic energy of the electrons in that region), and $d$ is the distance from the tip to the specimen. ${ }^{17}$ After passing through the specimen the electron travels to the detector, which in our apparatus can be negatively biased to decelerate the electrons. The time of flight for an electron for the specimen to detector region is given by

$T O F_{d e t}=\left(\frac{m_{e}(D-d)}{e V_{d e t}}\right)\left[\sqrt{\frac{2 e V_{t i p}}{m_{e}}}-\sqrt{\frac{2 e V_{t i p}}{m_{e}}-\frac{2 e V_{d e t}}{m_{e}}}\right]$,

where $D-d$ is the specimen to detector distance, $V_{\text {det }}$ is the retarding voltage place on the front of the detector. Once the electrons are detected a stop signal is sent to the TAC and the difference between the start and stop signals give the time of flight. For operation as a time of flight energy analyzer, Eq. (2) can be ignored because temporal dispersion in the tipto-specimen region is negligible compared to the specimento-detector region, see Fig. 3 (bottom). Typical time of flight data can be seen in Fig. 4. Data acquisition times were $10 \mathrm{~min}$, a retarding voltage of $10 \mathrm{~V}$ was put on the front of the detector, all data were taken with the same tip-to-specimen and the same specimen to detector distances. If each femtosecond laser pulse incident on the tip initiated electron emission, 


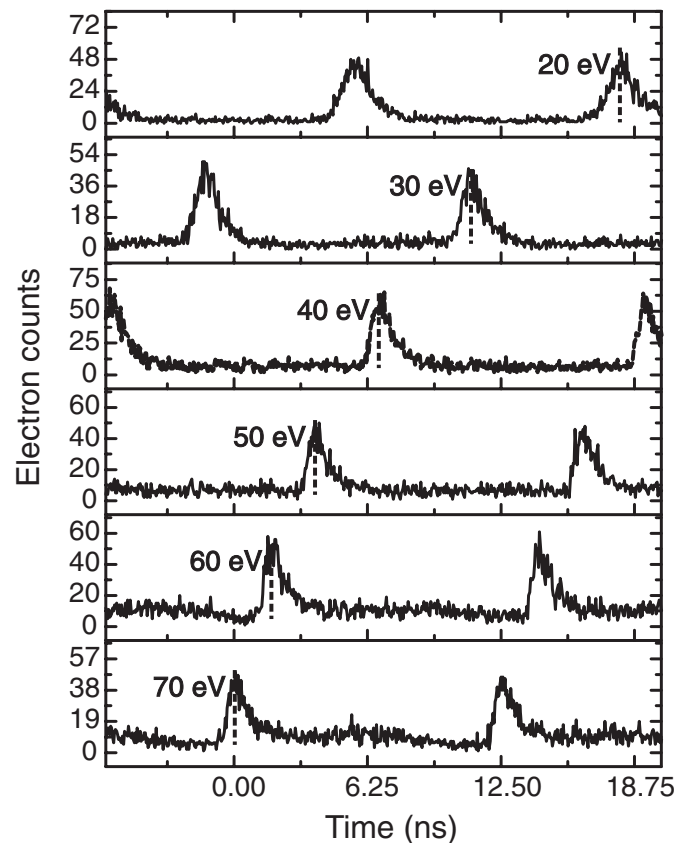

FIG. 4. Electron time of flight data. Plots are shown for 20, 30, 40, 50, 60, and $70 \mathrm{eV}$ electrons as labeled in the figure. The zero time point is not calibrated and each spectrum has multiple peaks because not every laser pulse from the $80 \mathrm{MHz}$ laser causes photoemission. The peaks shift to the right as the electron energy decreases due to the longer time of flight. Dashed lines are a guide to the eye for the center of each peak.

there would only be a single peak for each spectrum shown in Fig. 4. This would be due to the fact that for every "start" pulse to the TAC (which is triggered by the $80 \mathrm{MHz}$ from the laser), there would be an electron to hit the detector and provide the "stop" pulse. However, the data were taken when operating the pulsed electron source at a rate of much less than 1 electron per pulse (closer to $\sim 1000$ electrons/s), thus resulting in multiple peaks, all separated by the inverse of the repetition rate of the laser (12.5 ns). In addition as the tip voltage is decreased, the peak positions shift to the right as it takes a longer time for the electron to reach the detector. The zero time point on the horizontal access is not calibrated, but can be found by fitting the peaks from different energies to the above time of flight equations. ${ }^{16}$ At higher electron energies the temporal widths of the peaks are dominated by the resolution of the detector, when compared to increases in width due to temporal dispersion. At lower electron energies the peak widths begin to broaden because the electrons have both longer transit times to the detector and the $\Delta \mathrm{E}$ of the beam becomes large relative to the primary energy $\mathrm{E}$ of the beam. This causes the broadening of the temporal width of the peak due to dispersion of the packet to become discernible from the detector resolution. The temporal width of the peaks in the higher energy electron spectra converges to the detector resolution, because at the higher energies the temporal dispersion due to a finite $\Delta \mathrm{E}$ becomes negligible. After summing multiple peaks in the $20 \mathrm{eV}$ and $70 \mathrm{eV}$ scans, the full widths at half maximum using a Gaussian fit are found to be $1.6 \mathrm{~ns}$ and $1.0 \mathrm{~ns}$, respectively. The detector resolution is currently $1.00 \pm 0.05 \mathrm{~ns}$, which is found from the peaks at higher energy $(70 \mathrm{eV})$ and is mainly limited by the detector electronics used; however, other currently available electron detectors can provide resolutions of the order of $100 \mathrm{ps},{ }^{16}$ which in turn would provide a higher energy resolution. By using an energy spread of $1.0 \mathrm{eV}$ and convoluting with the detector resolution of $1.0 \mathrm{~ns}$, the width of the $20 \mathrm{eV}$ electron peak of $1.6 \mathrm{~ns}$ is calculated and is in close agreement to the $0.75 \mathrm{eV}$ width found in Ref 16 .

The time of flight energy spectrometer using photoelectrons provides additional flexibility to do spectroscopy of thin specimens with different electron energies on samples such as graphene or carbon nanotubes, when investigating the plasmon energy loss region. ${ }^{18}$

\section{DISCUSSION}

\section{A. Operation of photoelectron point projection microscope as an ultrafast electron microscope}

The design of this microscope has several advantages when used as point projection ultrafast electron microscopy (PPUEM). The first is that there are no lenses or crossovers, which reduce space charge effects on the temporal and spatial properties of the electron packets, even when only a few electrons are in the packet. The second advantage is that the curvature of the nanometer tip source creates a nonlinear electric field which accelerates the electrons quickly, while greatly decreasing the temporal dispersion of the electron packet as it propagates towards the specimen. A third advantage is that for magnifications of $\sim 1000 \times$ or greater, the tip-to-specimen distance $d$ is of the order of $\mu \mathrm{m}$, which means that the electron packet has very little chance to disperse before reaching the specimen.

For ultrafast electron microscopes that operate in the "single" electron mode, ${ }^{1}$ or one electron per packet, space charge can be ignored and the primary cause of temporal broadening is dispersion. Because each photoelectron emission event from the metal tip is statistical in nature, the electrons are "born" with a distribution of different energies. ${ }^{19}$ This causes an energy spread in the electron packets (even when containing less than a single electron), corresponding to different velocities, causing a broadening (or dispersion) of the average pulse duration while it propagates.

Typically, electron sources such as those implemented in ultrafast electron crystallography (UEC) as well as traditional transmission electron microscope based UEM's use a simple diode design, which creates a constant electric field with a maximum strength of a $10^{6} \mathrm{~V} / \mathrm{m}$, followed by a drift region to a specimen at electron energies of $10 \mathrm{keV}$ to hundreds of $\mathrm{keV}^{20}$ The time of flight of an electron (ignoring space charge) for these standard geometries can be approximated as

$$
T O F_{U E C / U E M}=d_{\text {accel }} \sqrt{\frac{2 m_{e}}{e V_{\text {accel }}}}+d_{d r i f t} \sqrt{\frac{m_{e}}{2 e V_{\text {accel }}}},
$$

where $V_{\text {accel }}$ is the accelerating voltage of the electrons, $d_{\text {accel }}$ is the accelerating plate separation, and $d_{\text {drift }}$ is the drift distance from the source to the specimen. Both Eq. (2) which describes the time of flight of an electron traveling from a tip-to-specimen in a PPUEM and Eq. (4) are rudimentary; neither takes into account initial transverse velocities of the electrons and thus should be treated as a lower limit on the 
dispersion. However, using Eqs. (2) and (4) comparisons can be made between the two different UEM designs and how dispersion in each affects the electron pulse duration. Using the equations the time of flight of a fast $(E+\Delta E / 2)$ and slow $(\mathrm{E}-\Delta \mathrm{E} / 2)$ electron can be calculated to find the electron pulse duration increase due to dispersion, where $\mathrm{E}$ is the central electron energy and $\Delta \mathrm{E}$ is the energy spread in the pulse. This estimation ignores the initiating laser pulse duration, which would need to be convolved with the pulse duration spread due to dispersion and is not valid for packets containing more than a single electron. The results for dispersion at different tip-to-specimen distances are compared for a traditional UEC/UEM design at $60 \mathrm{keV}$ versus a $25 \mathrm{eV}$ (100 nm tip), $250 \mathrm{eV}$ (100 nm tip), and a $2500 \mathrm{eV}$ (1000 nm tip) PPUEM and can be seen in Fig. 3 (bottom panel). At the $25 \mathrm{eV}$ with a tip radius of $100 \mathrm{~nm}$ there is a nearly zero $\mathrm{CW}$ emission of electrons due to the low electric field, which is given as $\mathrm{E}=\mathrm{V}_{\text {tip }} /\left(5 \mathrm{r}_{\text {tip }}\right)$ at the tip surface. Even at $250 \mathrm{eV}$ with the $100 \mathrm{~nm}$ tip the $\mathrm{CW}$ emission is negligible; however for a $100 \mathrm{~nm}$ it is impossible to reach $2500 \mathrm{eV}$ before the tip is destroyed due to excessively high fields. To keep the electric field the same at the tip surface, and hence the $\mathrm{CW}$ tunneling current negligible, the $2500 \mathrm{eV}$ plot in Fig. 3 (bottom panel) is modeled with a $1000 \mathrm{~nm}$ tip. What we find is that for very small tip-to-specimen distances the PPUEM has greatly decreased pulse dispersion when compared to more traditional UEM designs. A recently completed numerical study that models our exact experimental geometry supports the conclusion that this microscope when operated as a PPUEM can greatly decrease electron pulse broadening due to dispersion, especially as the tip-to-specimen distance is kept to the tensof- $\mu \mathrm{m}$ range. ${ }^{14}$ We note that time-varying electric fields, such as those in RF compressors/sources which have already been used in a complimentary femtosecond electron shadow imaging method, ${ }^{6}$ or optical fields ${ }^{12,13}$ could be used to further decrease electron packet durations.

The spatial resolution of the microscope is limited currently to $\sim 100 \mathrm{~nm}$, due to mechanical vibration of the microscope. With the addition of vibration isolation and an ion pump improved spatial resolution should be expected, and in principle there is nothing limiting the resolution from approaching that of a standard point projection microscope, which has been demonstrated to reach resolutions better than $\sim 10 \mathrm{~nm}$ at operating voltages in the hundreds of eV range. ${ }^{15}$ This spatial resolution combined with temporal resolutions of the order of $100 \mathrm{fs}$ would make the microscope capable of photon-induced near field electron microscopy (PINEM), which enables the imaging of evanescent near fields. ${ }^{21}$ Normally, electron energies below $1 \mathrm{keV}$ would be detrimental to imaging due to their insufficient energy to penetrate samples. ${ }^{22}$ However, electrons used in PINEM imaging need only to pass near the nanostructure and not through it making the "shadow" imaging with this microscope particularly useful. Spectrometry can be accomplished through time-of-flight energy analysis as discussed above or through a retarding field analyzer, allowing energy-specific images to be captured. ${ }^{16}$

The pump fluence needed to conduct PINEM imaging on nanostructures is of the order of $1 \mathrm{~mJ} / \mathrm{cm}^{2}$ or more which is the upper limit of what can be reached with a standard

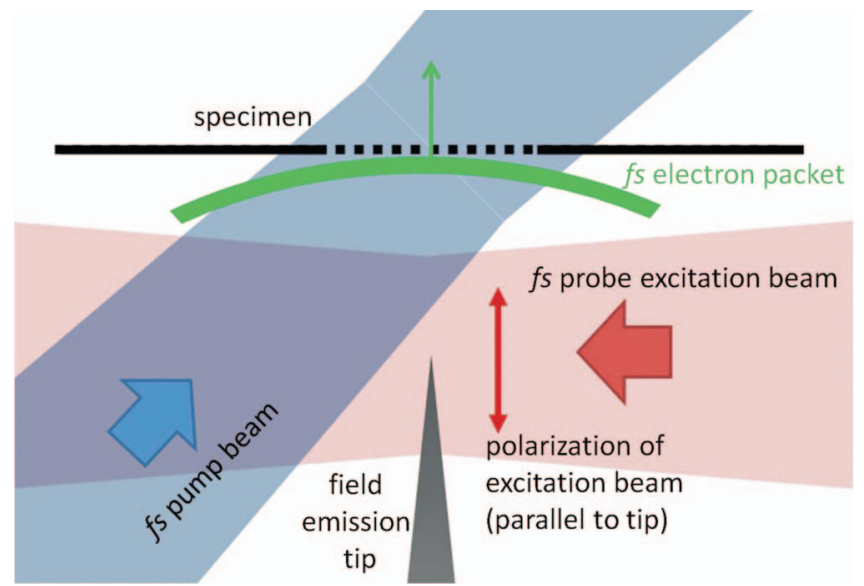

FIG. 5. Magnified 2D figure of the tip and specimen region while operating as an ultrafast electron microscope. The focus diameter for the blue pump pulse and the red probe excitation pulse are each $10 \mu \mathrm{m}$. The tip-to-specimen distance is also $10 \mu \mathrm{m}$. The red line with arrow points depicts the polarization of the probe excitation pulse parallel to the tip. The polarization of the pump beam should be made perpendicular to the tip to decrease the likelihood of it causing photoelectron emission. The green curved line shows the femtosecond electron pulse. The dashed black line represents a thin specimen placed in a $10 \mu \mathrm{m}$ diameter pinhole. The pinhole can facilitate alignment of the pump laser beam on the specimen by maximizing laser light throughput.

femtosecond oscillator. A more appropriate laser for UEM experiments, including those described below would be a commercially available $1 \mathrm{MHz}, 800 \mathrm{~nm}, 100 \mathrm{fs}$ with $\sim 1 \mu \mathrm{J}$ per pulse. Our microscope operated with this laser would still give sufficient electron signal when operating in "single" electron mode at $1 \mathrm{MHz}$ with $30 \mathrm{~s}$ imaging exposures, but would increase flexibility when pumping specimens due to its ability to create much higher fluences of $\sim 100 \mathrm{~mJ} / \mathrm{cm}^{2}$ at the specimen.

The focal size of the probe laser electron excitation pulse is the limiting factor for operating the photoelectron microscope as an UEM at higher magnifications, because as the tip is moved closer to the specimen the laser focus will overlap the specimen/holder, which will result in decreased electron emission from the tip apex. While the pump-probe geometry is shown in Fig. 1, a magnified image of the specimen region is shown in Fig. 5, which shows a tip-to-specimen distance of $10 \mu \mathrm{m}$, with pump and probe laser pulses each with a diameter of $10 \mu \mathrm{m}$. The pump and probe laser beams will enter the microscope from opposite sides of the microscope and by minimizing the spatial size of the specimen the probe beam can pass the specimen without hitting it, see Fig. 5. To facilitate alignment of the pump beam a $10 \mu \mathrm{m}$ pinhole is placed over the specimen and the pump beam transmission through the pinhole can be viewed from a side optical port on the vacuum system to optimize its spatial alignment on the specimen. Due to the pump beam beginning to impinge on the tip when tip-to-specimen distances are below $10 \mu \mathrm{m}$, this experimental region is shaded in Fig. 3 bottom, indicating geometries that are decreasingly accessible while operating as a UEM. As long as the pump beam polarization is kept perpendicular to the tip axis,,${ }^{9,10}$ and it does not hit the tip its effect on the photoemission current from the tip will be negligible, even at the above mentioned pump fluences for PINEM. 


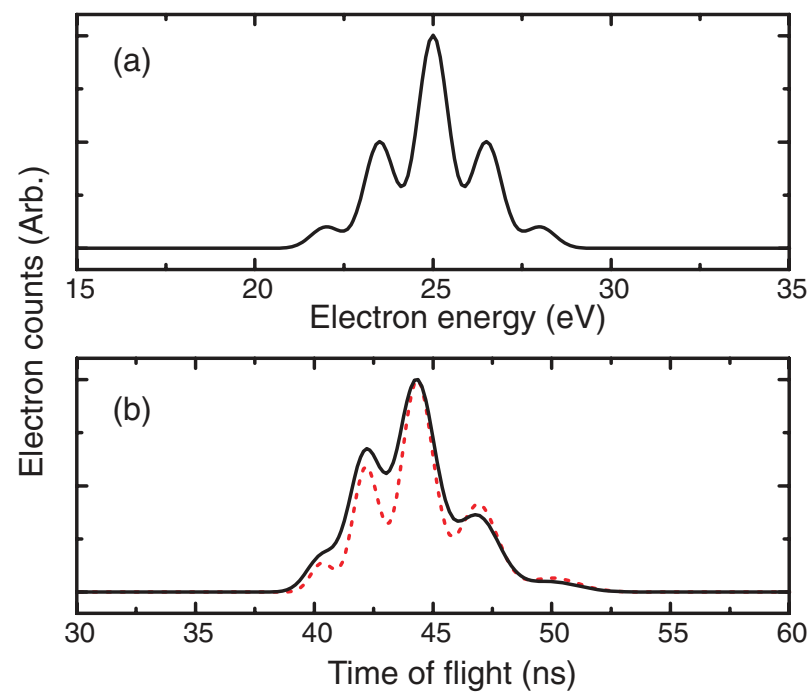

FIG. 6. Modeled energy distribution and time of flight energy spectrum for a $25 \mathrm{eV}$ electron pulse absorbing/emitting $\hbar \omega=1.5 \mathrm{eV}$ photons through the photon induced near field effect. Fig. 6(a) shows a modeled spectrum with sideband peaks that have absorbed/emitted $\mathrm{n}$ photons with weights of 0.5 for $\mathrm{n}= \pm 1$, and 0.1 for $\mathrm{n}= \pm 2$ with an energy spread of 1.0 eV. Fig. $6(\mathrm{~b})$ shows the same spectrum as it would be detected in our time of flight energy spectrometer. The dashed red curve shows the spectrum before convolution with the $1.0 \mathrm{~ns}$ detectors resolution and the solid line depicts the spectrum after convolution.

Magnification of $10 \mathrm{k}$ using a tip-to-specimen distance of $10 \mu \mathrm{m}$ will give sufficient resolution for PINEM imaging (of, for example, $100 \mathrm{~nm}$ diameter silver nanowires) and taking a temporal scan of $10 \mathrm{ps}$ with $100 \mathrm{fs}$ steps using $30 \mathrm{~s}$ imaging exposures would require a total scan of $50 \mathrm{~min}$, which is equivalent to the scan times used in other UEM experiments. $^{21}$

In addition to taking images at the different delay points, energy spectroscopy of the photon emission/absorption by the electron beam can be simultaneously collected at the same delay points with the time of flight of energy analyzer described above. To show the feasibility of spectroscopically resolving the emission/absorption with our time of flight energy analyzer we use Eq. (3) to model the predicted spectrum in Fig. 6. Figure 6 (top) shows the energy spectrum of a $25 \mathrm{eV}$ kinetic primary electron energy with sideband peaks of $\pm \mathrm{n} \hbar \omega$, with $\hbar \omega=1.5 \mathrm{eV}$ for an $800 \mathrm{~nm}$ pump beam. The spectrum is modeled with a series of Gaussian peaks with $1.0 \mathrm{eV}$ width, with relative intensities of 0.5 for $\mathrm{n}= \pm 1$, and 0.1 for $\mathrm{n}= \pm 2$ when compared to the $\mathrm{n}=0$ primary energy peak. This spectrum is equivalent to those presented in similar experiments conducted on surfaces, where a pump fluence of the order of tens of $\mathrm{mJ} / \mathrm{cm}^{2}$ is used. ${ }^{23}$ This energy spectrum, Fig. 6 (bottom), is modeled for the experimental situation when our microscope is operating at $-25 \mathrm{~V}_{\text {tip }}(25 \mathrm{eV}$ kinetic energy at specimen) with a retarding $\mathrm{V}_{\text {det }}$ of $17 \mathrm{~V}$. Converting the energy spectrum into time of flight spectrum for our microscope using Eq. (3), and then convoluting that spectrum with our $1.0 \mathrm{~ns}$ temporal resolution shows that the sideband peaks could be resolved. The time of flight spectrum is asymmetric because the velocities of the electrons are proportional to the square root of the kinetic energy, causing the pattern to spread out at lower energies. A particularly useful improvement to the apparatus would be the addition of a delay line electron detector which can provide spatial resolution equivalent to our current detector, but in addition can simultaneously provide arrival time information with $\sim 100$ ps resolution for each individual electron hit position. ${ }^{24}$ This would greatly expand the microscopes ability to take energy selective ultrafast images at each delay position.

\section{CONCLUSION}

We have shown that a point projection microscope combined with a femtosecond duration photoelectron source can spatially resolve objects on the $100 \mathrm{~nm}$ scale. This microscope can be used to image arbitrary specimens while simultaneously functioning as a time of flight electron energy analyzer. Using a simple model we also present modeled data that demonstrate the expected capability of this microscope when operated as an ultrafast electron microscope. We also show theoretically that by operating at close tip-to-specimen distances dispersion of the electron packet can be kept to 10 fs or less, which would be at least an order of magnitude improvement when compared to current stroboscopic UEM's. This microscope will be particularly useful as a tool to follow the temporal dynamics of excited evanescent optical fields near nanostructures with the PINEM technique. ${ }^{21}$ With the addition of a phase stabilized laser with pulses of only a few femtoseconds it may be possible to follow the evolution of plasmons in nanoparticles during laser excitation with nanometer spatial and sub-cycle temporal resolutions, ${ }^{25}$ and prospects for greatly increased electron degeneracy may allow the use of this microscope for the study of electron quantum optics. $^{26}$

\section{ACKNOWLEDGMENTS}

This work was supported by a Trinity College FRC grant and by a CT Space grant. A special thanks is given to Professor Ahmed Zewail and Caltech for the generous donation of the ultrafast laser system which made this research possible. The authors also thank Pratistha Shakya for her assistance in creating Figs. 1 and 4.

${ }^{1}$ A. H. Zewail, "Four-dimensional electron microscopy," Science 328(5975), 187-193 (2010)

${ }^{2}$ L. Piazza, D. J. Masiel, T. LaGrange, B. W. Reed, B. Barwick, and F. Carbone, "Design and implementation of a fs-resolved transmission electron microscope based on thermionic gun technology," Chem. Phys. 423, 79-84 (2013).

${ }^{3}$ R. K. Raman, Z. Tao, T.-R. Han, and C.-Y. Ruan, "Ultrafast imaging of photoelectron packets generated from graphite surface," Appl. Phys. Lett. 95(18), 181108 (2009).

${ }^{4}$ M. Centurion, P. Reckenthaeler, S. A. Trushin, F. Krausz, and E. E. Fill, "Picosecond electron deflectometry of optical-field ionized plasmas," Nat. Photon. 2(5), 315-318 (2008).

${ }^{5}$ J. Li, X. Wang, Z. Chen, R. Clinite, S. S. Mao, P. Zhu, Z. Sheng, J. Zhang, and J. Cao, "Ultrafast electron beam imaging of femtosecond laser-induced plasma dynamics," J. Appl. Phys. 107(8), 083305 (2010).

${ }^{6}$ C. M. Scoby, R. K. Li, E. Threlkeld, H. To, and P. Musumeci, "Singleshot 35 fs temporal resolution electron shadowgraphy," Appl. Phys. Lett. 102(2), 023506 (2013). 
${ }^{7}$ S. K. Sekatskii, S. V. Chekalin, A. L. Ivanov, V. O. Kompanets, Y. A. Matveets, A. G. Stepanov, and V. S. Letokhov, "Ultrahigh-spatialresolution photoelectron projection microscopy using femtosecond lasers," J. Exp. Theor. Phys. 88(5), 921-925 (1999).

${ }^{8}$ B. N. Mironov, S. A. Aseev, S. V. Chekalin, V. F. Ivanov, and O. L. Gribkova, "Laser photoelectron projection microscopy of an organic conducting polymer," JETP Lett. 92(11), 779-782 (2010).

${ }^{9}$ B. Barwick, C. Corder, J. Strohaber, N. Chandler-Smith, C. Uiterwaal, and H. Batelaan, "Laser-induced ultrafast electron emission from a field emission tip," New J. Phys. 9, 142 (2007).

${ }^{10}$ P. Hommelhoff, Y. Sortais, A. Aghajani-Talesh, and M. Kasevich, "Field emission tip as a nanometer source of free electron femtosecond pulses," Phys. Rev. Lett. 96, 077401 (2006).

${ }^{11}$ P. Hommelhoff, C. Kealhofer, and M. A. Kasevich, "Ultrafast electron pulses from a tungsten tip triggered by low-power femtosecond laser pulses," Phys. Rev. Lett. 97, 247402 (2006).

${ }^{12}$ C. Ropers, D. R. Solli, C. P. Schulz, C. Lienau, and T. Elsaesser, "Localized multiphoton emission of femtosecond electron pulses from metal nanotips," Phys. Rev. Lett. 98, 043907 (2007).

${ }^{13}$ M. Krüger, M. Schenk, and P. Hommelhoff, "Attosecond control of electrons emitted from a nanoscale metal tip," Nature (London) 475(7354), 78-81 (2011).

${ }^{14}$ A. Paarmann, M. Gulde, M. Müller, S. Schäfer, S. Schweda, M. Maiti, C. Xu, T. Hohage, F. Schenk, C. Ropers and R. Ernstorfer, "Coherent femtosecond low-energy single-electron pulses for time-resolved diffraction and imaging: A numerical study," J. Appl. Phys. 112(11), 113109 (2012).

${ }^{15}$ V. T. Binh, V. Semet, and N. Garcia, "Low-energy-electron diffraction by nano-objects in projection microscopy without magnetic shielding," Appl. Phys. Lett. 65(19), 2493-2495 (1994).
${ }^{16}$ S. A. Hilbert, B. Barwick, M. Fabrikant, C. Uiterwaal, and H. Batelaan, "A high repetition rate time-of-flight electron energy analyzer," Appl. Phys. Lett. 91(17), 173506 (2007).

${ }^{17}$ R. Gomer, Field Emission and Field Ionization (Harvard University Press, Cambridge, 1961).

${ }^{18}$ J.-Y. Park, S. H. Kim, Y. D. Suh, W. G. Park, and Y. Kuk, "Low-energy electron point source microscope with position-sensitive electron energy analyzer,” Rev. Sci. Instrum. 70(11), 4304-4307 (1999).

${ }^{19}$ M. Aidelsburger, F. O. Kirchner, F. Krausz, and P. Baum, "Single-electron pulses for ultrafast diffraction," Proc. Natl. Acad. Sci. U.S.A. 107(46), 19714-19719 (2010).

${ }^{20}$ A. Gahlmann, S. T. Park, and A. H. Zewail, "Ultrashort electron pulses for diffraction, crystallography and microscopy: Theoretical and experimental resolutions," Phys. Chem. Chem. Phys. 10, 2894-2909 (2008).

${ }^{21}$ B. Barwick, D. Flannigan, and A. H. Zewail, "Photon induced near-field electron microscopy," Nature (London) 462, 902-906 (2009).

${ }^{22}$ B. McMorran, J. D. Perreault, T. Savas, and A. Cronin, "Diffraction of 0.5 $\mathrm{keV}$ electrons from free-standing transmission gratings," Ultramicroscopy 106, 356-364 (2006).

${ }^{23}$ G. Saathoff, L. Miaja-Avila, M. Aeschlimann, M. M. Murnane, and H. C. Kapteyn, "Laser-assisted photoemission from surfaces," Phys. Rev. A 77(2), 022903 (2008).

${ }^{24}$ P. S. Kirchmann, L. Rettig, D. Nandi, U. Lipowski, M. Wolf, and U. Bovensiepen, "A time-of-flight spectrometer for angle-resolved detection of low energy electrons in two dimensions," Appl. Phys. A 91(2), 211-217 (2008).

${ }^{25}$ S. T. Park and A. H. Zewail, "Enhancing image contrast and slicing electron pulses in 4D near field electron microscopy," Chem. Phys. Lett. 521, 1-6 (2012).

${ }^{26}$ S. A. Hilbert, C. Uiterwaal, B. Barwick, H. Batelaan, and A. H. Zewail, "Temporal lenses for attosecond and femtosecond electron pulses," Proc. Natl. Acad. Sci. U.S.A. 106, 10558-10563 (2009). 